\title{
THE SEMANTICAL STRUCTURE OF LANGUAGE UNITS EXPRESSING THE LEVEL OF A SYMBOL
}

\author{
Nilufarkhon Ravshanovna Makhmudova \\ Teacher of Andizhan State University, Andizhan region, Republic of Uzbekistan
}

Article DOI: https://doi.org/10.36713/epra1683

DOI No: 10.36713/epra1683

\begin{abstract}
In the article, the semantic structure of language units representing the hierarchy of a character is analyzed in detail using data from the scientific literature. Besides, this work clarify the issue of the structure of the meanings of lexicalsemantic means (lexical-semantic indicators of graduality) representing gradation as well.
\end{abstract}

KEY WORDS: lexical layer, character, hierarchy, language units, semantic structure.

\section{INTRODUCTION}

The lexical layer is the main layer that represents the gradual category. In this regard, it is necessary to clarify the issue of the structure of the meanings of lexical-semantic means (lexicalsemantic indicators of graduality) representing gradation. Because the structure of this indicator determines its function in language and speech, its pragmatic nature, that is, its possibility of application in one or another type of discourse.

\section{METHODS}

This analysis is based on the theory of multicomponent structure of lexical meaning [1, p.19]: denotative-signifiable (D-S), connotative (K), grammatical $(\mathrm{G})$, sociolinguistic (S-L) [1, p.20 ] macrocomponents were isolated. Each of the listed macro components consists of a set of semaphores that provide the appropriate type of information.

\section{RESULTS AND DISCUSSIONS}

Lexical-semantic indicators of graduality are represented by LB, which fixes the gradation of the character in the text. In their semantic structure, as in the semantic structure of all words, the aforementioned macrocomponents are identified. According to the study, content macrocomponents have different ratios in different lexical-semantic indicators. One of the macrocomponents specific to the meanings of gradation indicators may be amplified and have the status of a key component, which affects its function in speech and determines its applicability in appropriate discourses.

Lexical-semantic indicators of graduality in terms of the ratio of denotative-signifiable and connotative macrocomponents are divided into two main groups: nominative indicators and connotative indicators.

The first group consists of lexical-semantic indicators, in which the denotative-signifiable macrocomponent is the main, and the connotative meaning is weakened. The meanings of these types of indicators perform a nominative function, in this respect the indicators are called nominative: very surprised, special attention, extreme poverty, much more enjoyable, a few seconds, a little excited: but because you have often heard me say so-was then a much larger and better place, and a much more valuable property than it is now. (Charles Dickens, The Barnaby rudge); "you are a little ruffled and out of humor. I'll wait till you're quite yourself again. I am in no hurry." (Charles Dickens, The Barnaby rudge)

In the group of nominative lexical-semantic indicators by function can be distinguished the following types:

- Indicators whose main task is to determine the degree (intensity, size, degree, etc.). The denotative-signifiable macrocomponent in the content of indicators is manifested only in gradual semaphores: completely different - completely 
different meaning, such serious contacts - such serious relationships. Indicators that do not have the main function of determining gradality. The denotative-signifiable macrocomponent has gradual semaphores, but they are not unique and primary. For example, the main function of grief $\mathrm{LB}$ is to determine the emotional state, but in the denotativesignifiable macro component of the content are also identified gradual semaphores that provide information about the greater degree of manifestation of the sign: grief = "pain, deep grief, mourning."Among the nominative lexical-semantic indicators of graduality, there are indicators in which both denotative-signifiable and connotative macrocomponents are expressed in meaning, with evaluative and gradual semaphores appearing as nuclear semaphores. The meanings of these indicators perform a nominative-evaluative function, in this regard we call them nominative-evaluative indicators of graduality: highly appreciated, fundamental difference, qualitively new level and etc.

Connotative lexical-semantic indicators of graduality include LB, which has both a denotativesignifiable and a connotative macro component, but the connotative macro component is the main one. The meanings of these indicators perform a connotative-descriptive function: scaryscandal - a terrible quarrel, astronomicalsum - a price in heaven, inhumanvanity - inhuman anger, rage.

Like nominative indicators, connotative indicators are divided into two groups according to their function:

1) Indicators whose main function is to determine graduality: terrible long break, monstrouspower - terrible power, cruelpunishment cruel punishment, scarynoise - horrible (ear-piercing) noise, sharplook - sharp gaze, colossalbuilding - huge building; aterriblylongpause, anunimaginablecrush.

2) Indicators that do not have a primary function of determining gradality: amazement, anger, love, noise, serenity, despair. For example, the main function of the word amazement is to define an emotional effect, although in the context of this word gradual semantics are also presented: to be surprised, = "to make a great impression".

According to the derivational type of lexical meaning, both nominative and connotative lexicalsemantic indicators of graduality can be divided into primitive and artificial types. The presence of gradual semaphores in the content of root-specific indicators cannot be explained at this stage of language development, as the internal form of these LBs is lost: quite stunned, very noticeable, completely different meaning, meticulous, frustrated, distracted (nominative indicators); rude people, overjoyed, great fight, open-mouthed, stunned, stubborn, stubborn, overly respectful, esteemed, victorious (connotative indicators). The existence of gradual semantics in the meanings of nominative- constructive and connotative-constructive indicators can be determined by the meanings they represent (semantic and word-forming). For example, gradual semaphores (destructive precipitation mixed with wind, rain, and snow) defined in the semantics of a storm horse, the word flood in semantic derivation includes the main nuclear semaphores in the sense of "something strong manifestation": the storm of perceptions.

The horror generated by word-formation from the horse is used to describe the "very strong" gradual semaphore. intensity semaphores become active, intensify, and take on the status of chief nuclear semaphores: terribly hot, terribly beautiful, terribly hungry. In speech, cases are identified in which gradual indicators reinforce occasional meanings. Gradual semaphores appear as peripheral semaphores in the sense that they form these indicators. In speech, they become more active and stronger, as a result of which the word acquires an occasional content, which is activated only in a certain speech situation: the teeth fall out and become "bald", and so on.

In the sense of connotative lexical-semantic indicators the following can be strengthened:

- Emotional semaphores: angry gestures, hopeless courage, earnest desire, insane speed, cruel laughter, brainwashing, staring, stunned, chubby child, dreaming, screaming, shivering with fear dr .;

- emotional-evaluative semantics: a) positive: mature professionals, brilliant success, blessed, beautiful place, extremely beautiful lines, luxurious place, great gift, excellent stage work, great organization; b) Negative: opponent, unpleasant, sad situation, fatal shooting, fatal outcome. In the nuclear part of the meanings, both denotative-signifative and connotative semantics appeared (the semantics of "rational evaluation", "character level" are denotative, emotional evaluation and emotional semantics are connotative). The meanings of indicators perform a connotative-evaluative function, in this respect we call them connotative-evaluative;

-imaginary semaphores: industrial giants, a storm of negative emotions: In our country, there are successful manufacturing enterprises established in cooperation with the famous German industrial giants - MAN, Claas, Knauf and others. Bilateral cooperation in the automotive industry is developing rapidly. (http://nv.chamber.uz/uz/news/3997); We continue to watch and listen while the giants of the industry are continuously rewarded by their minions ingovernment [2].

The meanings of connotative lexicalsemantic indicators can be enhanced by the combination of different types of semantics:

-rational-evaluative and emotionallyevaluating semaphores: masculine power (positive rational-evaluative sema and positive emotionalevaluative sema); victory (positive rational- 
evaluative and emotional-evaluative semantics): Philip gave him back the letter. He felt a thrill of pride in his triumph. He had got his own way, and he was satisfied. His will had gained a victory over the wills of others. (W.Somerset Maugham, Of Human bondage);

-emotive and rational-evaluative semaphores: being lazy from the heat (emotion semen "suffering", negative rational-evaluative sema); burning with joy (emotion of "satisfaction", positive-evaluative sema); suffering (emotion of "suffering", negative rational-evaluative sema);

-emotional, rational-evaluative and emotional-evaluative semaphores: rude candidates (emotive seismic "anger", negative rationalevaluative and emotional-evaluative semaphores); cruel hatred (emotional semaphore of "enmity", negative rational-evaluative and emotional-evaluative sema);

- figurative, rational-evaluative and emotional-evaluative semantics: magnificent hundred years (figurative semantics, positive-rationalevaluative and emotional-evaluative semantics); fairy-tale life (figurative semantics, positive rationalevaluative and emotional-evaluative semantics);

- figurative, emotional, emotional-evaluative and rational-evaluative semantics: exile (in the sense of "very difficult, painful situation, life" - figurative semantics, emotional semantics of "suffering", negative rational-evaluative and emotional-evaluative semantics); inhuman laws (figurative, emotional cruelty, negative emotional-evaluative and rationalevaluative semantics); venomous gaze (figurative semantics, "hostile" emotional semantics, negative emotional-evaluative and rational-evaluative semantics).

The considered connotative lexical-semantic indicators of graduality can be called expressive. Given the fact that the concept of expressiveness is interpreted differently by scientists [3], it should be noted that in this work expressiveness is understood as the compatibility of one of the connotative semaphores with the semaphore of "large degree of character gradation." Expressive semaphores can consist of emotive semaphores, emotionally evaluative, figurative semaphores, if they are presented with gradual semaphores (especially intensity semaphores). It should be noted that the degree of expressiveness may vary. The more types of connotative semaphores are amplified in harmony with the intensity semaphore in the connotative macrocomponent, the higher the level of expressiveness of the meaning of the lexical-semantic index. Lexical-semantic indicators of graduality are characterized by a minimal level of expressiveness, and their connotative macrocomponent is enhanced by the combination of intensity semaphores with a kind of semaphore: with unmotivated figurative semaphore the absence forms a peculiar non- ambiguity of the content, and this enhances expressiveness; with emotive sema (toddler - small chubby child, baby) and others. The average level of expressiveness is characteristic of lexical-semantic indicators, whose connotative macrocomponent consists of two types of intensity semaphores: emotional and emotional-evaluative sema (rude candidates), figurative and emotional-evaluative semaphores (magnificent century). It has lexicosemantic indicators for maximum level of expressiveness, the connotative macrocomponent of which is enhanced by the combination of intensity semaphores with three types of semaphores: figurative, emotional and emotional evaluative semaphores (inhuman laws).

When we consider the specifics of the macrocomponent of meanings specific to the lexicalsemantic indicators of graduality, it is possible to distinguish indicators with grammatical indicators. These include the LB, which represents the grammatical category of the level of comparison: the more serious the situation, the most beneficial, the most successful, the most important day, the lowest result.

In the macro component of lexical-semantic indicators of graduality can be presented semantics that provide information about the style, the possibility of using the word in certain discourses. In this regard, we highlight the stylistically defined indicators. These types of indicators may have the following characteristics:

- Literary style: so much, so much, really. In this group, lofty words are singled out: to dream, to honor (carry in the head), to grieve hard: Salim ota Bianada, who once dreamed of the loss of oppressive rulers by a people who lived by honest labor, was uplifted by this dream. (Pirimqul Kadyrov, Humoyun and Akbar). These types of indicators are used in mass communication discourses;

-Speech style: among the indicators that are distinguished by an exaggerated, insidious, throwing style of speech, there are words that are typical of ordinary vernacular: - Lord, these words are a slander against a strong figure like you! (Pirimqul Kadyrov, Humoyun and Akbar); as far as power is concerned, at least, sal-pal, this type of very verbal indicator is used in informal communication discourses that do not obey the boundaries of etiquette. Anwar tried his best not to give away the secret, even though he was filled with this poisonous word like a snake. (Abdullah Qadiri, Scorpion from the Altar); Put it down! I'm totally back from you. I washed my hands and hit my armpits! Holmurod waved. Then he looked at Hakimov with hope and continued in a sad tone. Let me look at someone's husband in the eyes of this man, let me be a liar ... (Said Ahmad, The Sun). 


\section{CONCLUSION}

Thus, in the meanings of lexical semantic indicators are denotative-signifiable, connotative, grammatical and sociolinguistic macrocomponents. Nominative and connotative lexical-semantic indicators of graduality are distinguished according to the ratio of denotative-signifiable and connotative macro-components of meaning. According to their function, nominative and connotative indicators are divided into two groups: 1) indicators whose main function is to determine graduality, and 2) indicators whose main function is not to determine graduality. According to the derivational type of content, nominative and connotative indicators are divided into artificial and non-artificial indicators. Connotative indicators are characterized by minimum, medium, and maximum levels of expressiveness depending on the type of connotative semantics, which are presented in conjunction with graduality (intensity) semantics in the sense of indicators. Lexical-semantic indicators of grammatically and stylistically marked graduality are also distinguished by the specificity of the function.

\section{REFERENCES}

1. Pelepeichenko L.N. Transitional types of meanings of words (based on the material of the Russian language). - Kharkiv: KhGPU, 1994. P.19-20

2. https://www.huffpost.com/entry/how-ourleadership-canso_b_825168

3. Pelepeichenko L.N. Transitional types of meanings of words (based on the material of the Russian language). - Kharkiv: KhGPU, 1994. P.138. 